eines Inversen,

$$
\partial_{b}^{a} x \cdot \partial_{x}^{a} b=1
$$

bei zyklischer Vertauschung gilt

$$
\partial_{b}^{a} c \cdot \partial_{a}^{c} b \cdot \partial_{c}^{b} a=-1 .
$$

(Die ,Entartung“ “ ${ }_{a}^{a}$ ist in diesem Formalismus ein Symbol ohne Sinn, da die Art des Grenzüberganges nicht ersichtlich ist.) Für den Übergang zu einem anderen Koordinatennetz $(x, y) \rightarrow(a, b)$ wenden wir den ersten Teil von (A.1) auf $a$ und $b$ an, setzen die so erhaltenen Ausdrücke für $\mathrm{d} a(x, y)$ und $\mathrm{d} b(x, y)$ in den zweiten Teil von (A.1) ein und erhalten durch Koeffizientenvergleich aus (A.1) die Substitutionsregeln

$$
\partial_{x}^{y}=\partial_{x}^{y} a \partial_{a}^{b}+\partial_{x}^{y} b \partial_{b}^{a}
$$

und eine analoge Gleichung, in der $x$ und $y$ vertauscht erscheinen. Identifiziert man hier $a$ mit $x$, also $(x, y) \rightarrow(x, b)$, so ergibt sich wegen (A. 3) die ,,Substitutionsregel für die festzuhaltende Variable"

$$
\partial_{x}^{y}=\partial_{x}^{b}+\partial_{x}^{y} b \partial_{y}^{x} .
$$

wird stattdessen analog in (A.6) $a$ mit $y$ identifiziert, so folgt wegen (A.2) die ,eindimensionale Kettenregel" (Substitutionsregel für die Ableitungsvariable)

$$
\partial_{x}^{y}=\partial_{x}^{y} b \partial_{b}^{y}
$$

die auf $x$ angewandt gerade die Inversionsregel (A.4) beweist. Wird (A.7) auf $y$ angewendet, so ist wegen (A. 4) und (A. 2) auch die Vertauschungsregel (A. 5) bewiesen. Anwendung von $\partial_{x}^{y}$ auf $x$ ergibt wegen (A. 6) schließlich die symmetrische Beziehung

$$
\partial_{x}^{y} a \partial_{a}^{b} x+\partial_{x}^{y} b \partial_{b}^{a} x=1 .
$$

24 Nach H. A. BuchdahL, The Concepts of Classical Thermodynamics. At the University Press, Cambridge 1966, $\S 80$, S. 165 .

25 Für diesen Hinweis danke ich Herrn Dr. R. GöвEL.

\title{
Ein einfaches statistisches Modell für Transportvorgänge mit beschränkter Ausbreitungsgeschwindigkeit. I.
}

\author{
J. U. KELLER \\ Institut für Theoretische Physik der Rheinisch-Westfälischen Technischen Hochschule Aachen \\ (Z. Naturforsch. 25 a, 1202-1206 [1970]; eingegangen am 23. Mai 1970)

\begin{abstract}
A simple statistical model for transport-processes in material systems like heat conduction, diffusion and Brownian motion is given. The model consists of a one-dimensional unrestricted random walk process. The jump-probabilities of the random-walk-particle generally depend on the history of the particle. The transport equations following from this model describe, contrary to the equations given by Fick, Fourier, Smoluchowski and Fokker-Planck always transport-processes with bounded velocity of propagation.
\end{abstract}

\section{Einleitung}

Ein einfaches statistisches Modell für Transportvorgänge in Materie wie z. B. Wärmeleitung, Diffusion oder Brownsche Bewegung ist der klassische Irrflug-Proze ${ }^{1}$.

Dieses Modell besteht im einfachsten Fall aus einem oder vielen Teilchen, welche unabhängig voneinander jeweils zu den Zeitpunkten

$$
N \tau \quad(N=0,1,2, \ldots, \quad \tau=\text { const })
$$

Sonderdruckanforderungen an Dr. J. U. KelleR, Institut für Theoret. Physik der Technischen Hochschule Aachen, D-5100 Aachen, Templergraben 55. auf einem unbeschränkten eindimensionalen Gitter mit den Plätzen $n a \quad(n=0, \pm 1, \pm 2, \ldots, a=$ const $)$ statistisch verteilte Sprünge ausführen. Um von dieser mikroskopischen zu einer makroskopischen Beschreibung zu gelangen, muß man vom diskreten Modell zum Kontinuum übergehen:

$$
\begin{gathered}
a \rightarrow 0, \quad \tau \rightarrow 0 ; \quad N \rightarrow \infty, \quad n \rightarrow \infty, \\
\quad n a=x, \quad N \tau=t .
\end{gathered}
$$

Hier bedeuten $x$ und $t$ makroskopische Orts- und Zeitparameter. Aus dem Satz von der totalen Wahr-

1 S. Chandrasekhar, Rev. Mod. Phys. 15, 1 [1943]. - M. KAC, in: Selected Papers on Noise and Stochastic Processes, N. WaX (Ed.), Dover, N. Y. 1954, S. 295. - A. H. ReIF, Fundamentals of Statistical and Thermal Physics, McGraw-Hill, New York 1969, Chap. 1. 
scheinlichkeit (STW) ${ }^{2}$, formuliert für das diskrete mikroskopische Modell, folgen beim Grenzprozeß (E.1) makroskopische Transportgleichungen. Diese Gleichungen sind natürlich nur solange brauchbar, solange man makroskopisch von den stets vorhandenen Schwankungen ${ }^{3}$ absehen kann. Die Struktur dieser Transportgleichungen hängt nun entscheidend von der Art und Weise ab, wie der Grenzprozeß (E.1) durchgeführt wird. In der Literatur scheint bis heute nur der durch die Forderung

$$
\lim \left(a^{2} / \tau\right)=2 D=\text { const }
$$

mathematisch präzisierte Grenzprozeß (E.1) verwendet worden zu sein ${ }^{1,4}$. Dieser Einstein-Smoluchowski-Limes führt im Falle Brownscher Bewegung auf die Smoluchowski- oder Fokker-Planck-Gleichung ${ }^{1,4,5}$, auf das 2. Ficksche Gesetz im Falle von Diffusion und auf die Fouriersche Wärmeleitungsgleichung, wenn der Irrflug-Prozeß als Modell für Wärmeleitung angesehen wird ${ }^{5}$ (S. 291). Dieser Grenzprozeß führt aber zu einer prinzipiellen Schwierigkeit: Die Geschwindigkeit $(a / \tau)$, mit welcher das Irrflug-Teilchen (IT) seine Zitterbewegung ausführt, divergiert ${ }^{6}$ !

Dies spiegelt sich auch in der Smoluchowski-, Diffusions- bzw. Wärmeleitungsgleichung wider: Diese Gleichungen beschreiben Transportprozesse mit unbeschränkter Ausbreitungsgeschwindigkeit ${ }^{7}$. Dieser Befund widerspricht der speziellen Relativitätstheorie. Man weiß ferner aus dem Experiment, daß die Ausbreitungsgeschwindigkeit der obengenannten Transport-Prozesse höchstens von der Größenordnung der Schallgeschwindigkeit in dem betreffenden Medium ist. Der Einstein-Smoluchowski-Limes ist also in physikalischer Hinsicht unbefriedigend. Man könnte nun versuchen, den Grenzprozeß (E.1) nicht mit (E.2), sondern mit der Forderung

$$
\lim (a / \tau)=c=\text { const }
$$

2 W. Feller, An Introduction to Probability Theory and its Application, 3-rd Ed., J. Wiley \& Sons, New York 1952, S. 20.

3 L. D. Landau u. E. M. Lifschitz, Lehrbuch der Theoretischen Physik, Akademie Verlag, Berlin 1956, Bd. VI, S. $607 \mathrm{ff}$.

${ }^{4}$ M. Smoluchowski, Ann. Phys. 21, 756 [196]; 25, 205 [1908]; Phys. Z. 16, 321 [1915] ; 17, 557, 585 [1916]. A. Einstein, Ann. Phys. 17, 549, 559 [1905]; 19, 371, 380 [1906]. - D. R. Cox u. H. D. Miller, The Theory of Stochastic Processes, J. Wiley \& oSns, New York 1965, S. $46 \mathrm{ff}$. - A. Karlin, A First Course in Stochastic Processes, Academic Press, New York 1968, S. 273. - G. E. UhlenBeCK u. L. S. Ornstein, Phys. Rev. 36, 823 [1930]. - C. auszuwerten. Tut man das mit den in ${ }^{1,4}$ verwendeten Irrflug-Prozessen, so erhält man als Transportgleichung eine Wellengleichung 1. Ordnung, deren Lösungen ungedämpfte Wellen sind. Diese Gleichungen sind also zur Beschreibung dissipativer Prozesse nicht geeignet.

Es zeigt sich nun, daß diese Schwierigkeit im Limes (E.1) mit (E.3) dadurch bedingt ist, daß der allen Irrflug-Prozessen in ${ }^{1,4}$ zugrunde liegende statistische Prozeß eine Markoff-Kette 1. Stufe ist ${ }^{2}$ (S. 307), (Cox, Karlin) ${ }^{4}$. Dies bedeutet in physikalischer Hinsicht, daß beim Grenzübergang vom diskreten System zum Kontinuum (E.1), (E.3) die Trägheit des IT nicht berücksichtigt werden kann.

Es liegt nun nahe, zu versuchen, die im Limes (E.1), (E.3) auftretende Schwierigkeit dadurch zu überwinden, daß man den Irrflug-Prozeß durch eine Markoff-Kette höherer Stufe beschreibt ${ }^{8,9}$, daß man also die Vorgeschichte des IT stärker als bisher berücksichtigt. Dies ist tatsächlich möglich. Wir nehmen an, daß der dem Irrflug-Prozeß zugrunde liegende statistische Prozeß eine Markoff-Kette 2. Stufe ist, berücksichtigen also im Gegensatz zur 1. Markoff-Stufe die Trägheit des IT. Dann kann man einen physikalisch leicht interpretierbaren Grenzprozeß von Typ (E.1), (E.3) angeben, bei welchem aus dem STW für den diskreten Prozeß eine Transportgleichung vom Typ der Telegraphengleichung folgt:

$$
\tau \partial_{t}^{2} p+\partial_{t} p=b \partial_{x} p+D \partial_{x}^{2} p .
$$

Hierbei ist $p=p\left(\begin{array}{l}x \\ t\end{array}\right)$ die lokale Dichte der Aufenthaltswahrscheinlichkeit des Brownschen Teilchens bzw. die Dichte der diffundierenden Teilchen oder die Temperatur am Ort $x$ zur Zeit $t$. Ferner bedeuten: $\tau>0 \ldots$ Relaxationszeit, $b \ldots$ Beweglichkeit, $D$... Diffusionskonstante bzw. Temperaturleitfähigkeit $\left(\partial_{x}=\partial / \partial_{x}, \partial_{t}=\partial / \partial t\right)$. Die Gl. (E.4) beschreibt wegen $\tau>0$ Transportprozesse mit beschränkter

E. Wang u. G. E. Uhlenbeck, Rev. Mod. Phys. 17, 323 [1945]. - E. Nelson, Dynamical Theory of Brownian Motion, University Press, Princeton 1967.

5 R. BECKer, Theorie der Wärme, Springer-Verlag, Berlin 1964, S. 275.

${ }^{6}$ Die Bahnkurve der IT ist fast überal stetig und fast nirgendwo differenzierbar.

7 Bezüglich einer Untersuchung dieses Paradoxons im Rahmen der klassischen Thermodynamik der irreversiblen Prozesse sei auf I. MÜLER, Z. Phys. 198, 329 [1967] verwiesen.

8 J. L. Dоов, Stochastic Processes, J. Wiley \& Sons, New York 1953, S. 171, 185 ff.

9 J. Keller, Z. Physik 204, 47 [1967] ; 210, 142 [1968]. 
Ausbreitungsgeschwindigkeit. Dies folgt am einfachsten aus der Dispersions-Relation dieser Gleichung. Es ist das Ziel der vorliegenden Arbeit, die Gl. (E.4) mit Hilfe eines Irrflug-Prozesses der 2. Markoff-Stufe zu begründen.

\section{Irrflug-Prozesse der 2. Markoff-Stufe}

Wir führen zunächst gewisse Bezeichnungen ein und geben einige allgemeine Beziehungen an:

$p\left(\begin{array}{ll}m & n \\ M & N\end{array}\right)$

$\ldots m, n=0, \pm 1, \pm 2, \ldots ; \quad M, N=0,1,2, \ldots$

Simultane Wahrscheinlichkeit (SW) dafür, daß sich das IT sowohl zur Zeit $M \tau$ im Gitterpunkt $m a$ als auch zur Zeit $N \tau$ im Gitterpunkt $n a$ befindet.

$p\left(\begin{array}{c}\frac{a}{\tau} k \\ N+\frac{1}{2}\end{array}\right)$

... Wahrscheinlichkeit dafür, daß das IT zur Zeit $\left(N+\frac{1}{2}\right) \tau$ die Geschwindigkeit $(a / \tau) k$ besitzt.

$p\left(\begin{array}{ll|l}m & n & o \\ M & N & O\end{array}\right)$

...Bedingte Wahrscheinlichkeit (BW) dafür, daß sich das IT zur Zeit $o \tau$ im Gitterpunkt $o a$ befindet, wenn es sich zur Zeit $M \tau$ im Gitterpunkt $m a$ und zur Zeit $N \tau$ im Gitterpukt $n a$ befunden hat.

$p\left(\begin{array}{c|c}(a / \tau) k & (a / \tau) l \\ N-\frac{1}{2} & N+\frac{1}{2}\end{array}\right)$

... BW dafür, daß das IT zur Zeit $\left(N+\frac{1}{2}\right) \tau$ die Geschwindigkeit $(a / \tau) l$ besitzt, wenn es zur Zeit $\left(N-\frac{1}{2}\right) \tau$ die Geschwindigkeit $(a / \tau) k$ gehabt hat.

\section{Normierungen:}

$$
\begin{gathered}
\sum_{m, n=-\infty}^{\infty} p\left(\begin{array}{ll}
m & n \\
M & N
\end{array}\right)=1 \ldots \text { alle } M, N ; \quad(1 \mathrm{a}) \\
\sum_{o=-\infty}^{\infty} p\left(\begin{array}{cc|c}
M & N & o \\
m & n & O
\end{array}\right)=1 \ldots \text { alle } \underset{m, n}{M, O} .
\end{gathered}
$$

Diskretes Analogon zum Wahrscheinlichkeitsstrom am Ort $n a$ zur Zeit $N \tau$ :

$$
\begin{gathered}
j\left(\begin{array}{l}
n \\
N
\end{array}\right)=\frac{a}{2 \tau}\left(\pi_{a}\left(\begin{array}{l}
n \\
N
\end{array}\right)+\pi_{r}\left(\begin{array}{l}
n \\
N
\end{array}\right)\right), \\
\pi_{a}\left(\begin{array}{l}
n \\
N
\end{array}\right)=\sum_{i=-\infty}^{\infty} i p\left(\begin{array}{cc}
n & n+i \\
N & N+1
\end{array}\right),
\end{gathered}
$$

$$
\pi_{r}\left(\begin{array}{c}
n \\
N
\end{array}\right)=\sum_{i=-\infty}^{\infty} i p\left(\begin{array}{cc}
n-i & n \\
N-1 & N
\end{array}\right) .
$$

Ein über diskreten Orts- und Zeitparameter definierter stochastischer Prozeß heißt Markoff-Kette $s$. Stufe, wenn für alle höheren bedingten Wahrscheinlichkeiten $(r \geqq s)$ gilt:

$$
\begin{aligned}
& p\left(\begin{array}{ccc|c}
n_{r} & \ldots & n_{1} & n_{0} \\
N-r & N-1 & N
\end{array}\right)=p\left(\begin{array}{ccc|c}
n_{s} & \ldots & n_{1} & n_{0} \\
N-1 & N-s & N
\end{array}\right) \\
& \ldots \text { alle } n_{i}, i=0, \ldots, r, s=0,1,2, \ldots \text {; } \\
& N, r=0,1,2 \ldots r \leqq N .
\end{aligned}
$$

Der STW lautet für Markoff-Ketten 2. Stufe

$p\left(\begin{array}{cc}n & m \\ N & N+1\end{array}\right)=\sum_{l=-\infty}^{\infty} p\left(\begin{array}{cc}l & n \\ N-1 & N\end{array}\right) p\left(\begin{array}{cc|c}l & n & m \\ N-1 & N & N+1\end{array}\right)$.

Kennt man die BW der Kette, so lassen sich bei gegebener Anfangsverteilung $p\left(\begin{array}{ll}n & m \\ 0 & 1\end{array}\right)$ alle $\mathrm{SW}$ zu späteren Zeitpunkten aus (5) berechnen. Die BW der Kette können entweder theoretisch aus einem quantenmechanischen Modell für den betrachteten Transportprozeß berechnet werden, oder aber durch Beobachtung geeigneter Größen wie z. B. Mittelwert der Lage und Geschwindigkeit oder der Streuung des IT experimentell bestimmt werden.

Wir betrachten nun einen Irrflug-Prozeß der 2 . Markoff-Stufe im unbeschränkten eindimensionalen Gitter. Der Prozeß sei homogen in Ort und Zeit. Wir nehmen an, daß das IT nur Einzelsprünge, keine Multisprünge ausführen kann und daß es ferner keine Möglichkeit besitzt, an einem Ort zu verweilen. Für die BW kann dann im Hinblick auf (1 b) folgender Ansatz gemacht werden:

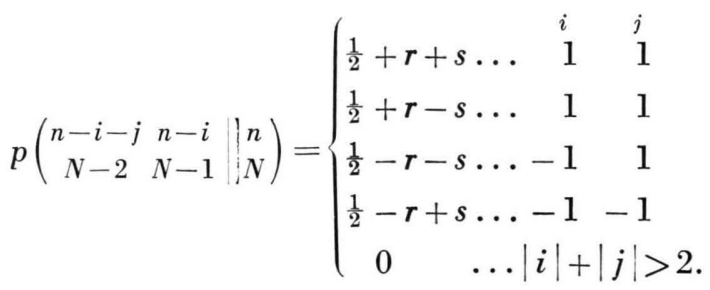

Die Dynamik der Kette wird durch 2 Parameter $r, s$ vollständig bestimmt. Diese Parameter können noch von $a, \tau$, aber nicht mehr von $n$ oder $N$ abhängen.

Ist $s=0$, so geht die Kette 2. Stufe in eine Markoff-Kette 1. Stufe über. Ist $r=0$, so sind die BW von spiegelsymmetrischen Sprungfolgen einander gleich. Wir sehen daher $r$ als Parameter einer äußeren Kraft an. Nach (6) gilt

$$
|r|+|s| \leqq \frac{1}{2} .
$$


Der STW liefert mit (6) die Rekursionen

$$
\begin{aligned}
& p\left(\begin{array}{cc}
n-1 & n \\
N & N+1
\end{array}\right)=\left(\frac{1}{2}+r+s\right) p\left(\begin{array}{cc}
n-2 & N-1 \\
N-1 & N
\end{array}\right) \\
& +\left(\frac{1}{2}+r-s\right) p\left(\begin{array}{cc}
n & n-1 \\
N-1 & N
\end{array}\right), \\
& p\left(\begin{array}{cc}
n+1 & n \\
N & N+1
\end{array}\right)=\left(\frac{1}{2}-r-s\right) p\left(\begin{array}{cc}
n & n+1 \\
N-1 & N
\end{array}\right) \\
& +\left(\frac{1}{2}-r+s\right) p\left(\begin{array}{cc}
n+2 & n+1 \\
N-1 & N
\end{array}\right) \text {. }
\end{aligned}
$$

Daraus ergeben sich mit $(3 \mathrm{a}, \mathrm{b})$ die Beziehungen

$$
\begin{aligned}
& 2 p\left(\begin{array}{c}
n \\
N+1
\end{array}\right)=(1+2 r) p\left(\begin{array}{c}
n-1 \\
N
\end{array}\right) \\
& +(1-2 r) p\left(\begin{array}{c}
n+1 \\
N
\end{array}\right)+2 s\left[\pi_{r}\left(\begin{array}{c}
n \\
N+1
\end{array}\right)-\pi_{r}\left(\begin{array}{c}
n-1 \\
N
\end{array}\right)\right] \\
& 2 \pi_{r}\left(\begin{array}{c}
n \\
N+1
\end{array}\right)=-(1-2 r) p\left(\begin{array}{c}
n+1 \\
N
\end{array}\right) \\
& +(1+2 r) p\left(\begin{array}{c}
n-1 \\
N
\end{array}\right)+2 s\left[\pi_{r}\left(\begin{array}{c}
n+1 \\
N
\end{array}\right)+\pi_{r}\left(\begin{array}{c}
n-1 \\
N
\end{array}\right)\right] \text {, } \\
& \pi_{a}(N+1)=(r+s)\left[\pi_{a}\left(\begin{array}{c}
n-1 \\
N
\end{array}\right)+p\left(\begin{array}{c}
n-1 \\
N
\end{array}\right)\right] \\
& +(r-s)\left[p\left(\begin{array}{c}
n+1 \\
N
\end{array}\right)-\pi_{a}\left(\begin{array}{c}
n+1 \\
N
\end{array}\right)\right] \text {. }
\end{aligned}
$$

Nach (7) gilt $\quad\left|\varrho_{0}\right|+\left|\sigma_{0}\right| \leqq \frac{1}{2}$.

Aus (9) folgt mit (10) :

Als Vorbereitung für den Grenzübergang zum Kontinuum führen wir folgende Bezeichnungen ein:

$$
\begin{gathered}
a=\Delta x, \quad \tau=\Delta t, \\
p\left(\begin{array}{c}
n \\
N
\end{array}\right) \rightarrow p\left(\begin{array}{c}
x \\
t
\end{array}\right), \quad p\left(\begin{array}{c}
a / \tau \\
N
\end{array}\right) \rightarrow p\left(\begin{array}{c}
\Delta x / \Delta t \\
t
\end{array}\right), \\
p\left(\begin{array}{c}
n \pm 1 \\
n+1
\end{array}\right) \rightarrow p\left(\begin{array}{c}
x \pm \Delta x \\
t+\Delta t
\end{array}\right), \\
\pi_{i}\left(\begin{array}{c}
n \\
N
\end{array}\right) \rightarrow \pi_{i}\left(\begin{array}{c}
x \\
t
\end{array}, \begin{array}{c}
\Delta x \\
\Delta t
\end{array}\right) \quad i=a, r, \\
z(a, \tau)=z(\Delta x, \Delta t), \quad z=r, s, \\
O\left((\Delta x)^{n}\right)=O_{x}{ }^{n}, \quad O\left((\Delta t)^{n}\right)=O_{t}{ }^{n} .
\end{gathered}
$$

Die Parameter $r, s$ mögen nach $\Delta x, \Delta t$ entwickelbar sein:

$$
\begin{aligned}
& r(\Delta x, \Delta t(\Delta x))=\varrho_{0}+\varrho_{1} \Delta x+O_{x}{ }^{2}, \\
& s(\Delta x, \Delta t(\Delta x))=\sigma_{0}+\sigma_{1} \Delta x+O_{x}{ }^{2} .
\end{aligned}
$$

$$
\partial_{t} p\left(\begin{array}{c}
x \\
t
\end{array}\right) \Delta t+O_{t}^{2}=\left[-2 r \Delta x \partial_{x}+\frac{1}{2}(\Delta x)^{2} \partial_{x}^{2}\right] p\left(\begin{array}{c}
x \\
t
\end{array}\right)-2 s \Delta x \partial_{x} \pi_{r}\left(\begin{array}{cc}
x & \Delta x \\
t & \Delta t
\end{array}\right)+O_{x}^{3},
$$

$$
\left(1+\Delta t \partial_{t}\right) \pi_{r}\left(\begin{array}{cc}
x & \Delta t \\
t & \Delta x
\end{array}\right)+O_{t}^{2}=\left[2 r-\Delta x \partial_{x}+r(\Delta x)^{2} \partial_{x}{ }^{2}\right] p\left(\begin{array}{c}
x \\
t
\end{array}\right)+s\left(2+(\Delta x)^{2} \partial_{x}^{2}\right) \pi_{r}\left(\begin{array}{c}
x \\
t
\end{array}, \begin{array}{c}
\Delta x \\
\Delta t
\end{array}\right)+O_{x}^{3},
$$

$$
\begin{aligned}
& \left(1+\Delta t \partial_{t}\right) \pi_{a}\left(\begin{array}{c}
x \\
t
\end{array}, \begin{array}{l}
\Delta t \\
\Delta x
\end{array}\right)+O_{t}{ }^{2}=\left(2 s-2 r \Delta x \partial_{x}+s(\Delta x)^{2} \partial_{x}{ }^{2}\right) \pi_{a}\left(\begin{array}{ll}
x & \Delta t \\
t & \Delta x
\end{array}\right) \\
& +\left(2 r-2 s \Delta x \partial_{x}+r(\Delta x)^{2} \partial_{x}^{2}\right) p\left(\begin{array}{l}
x \\
t
\end{array}\right)+O_{x}^{3} \text {. }
\end{aligned}
$$

Der Grenzübergang zum Kontinuum $\Delta x \rightarrow 0, \Delta t \rightarrow 0$ wird nun unter folgenden Voraussetzungen durchgeführt:

$$
\text { 1. Wir setzen } \Delta x=c \Delta t \text {, }
$$

wo $c>0$ eine Konstante ist. Diese Bedingung garantiert, daß die Geschwindigkeit, mit welcher das IT im kontinuierlichen Grenzfall seine Zitterbewegung ausführt, endlich ist.

2. Die Grenzwerte

$$
\lim _{\Delta t \rightarrow 0} \pi_{i}\left(\begin{array}{cc}
x & \Delta x \\
t
\end{array}, \Delta t\right)=\pi_{i}\left(\begin{array}{l}
x \\
t
\end{array}\right), \quad i=a, r
$$

sollen gleichmäßig für alle $x$ und $t$ existieren und in diesen Variablen in Taylor-Reihen entwickelbar sein. Diese Forderungen sind notwendig und hinreichend dafür, daß im kontinuierlichen Grenzfall an jedem Ort $x$ und zu jeder Zeit $t \geqq 0$ ein eindeutiger Wahrscheinlichkeitsstrom (bzw. Diffusions- oder
Wärmestrom) existiert.

3 . Wir setzen voraus, daß in (11)

$$
\sigma_{0}=\frac{1}{2}
$$

ist. Aus (6), (12), (16) folgt mit der Bezeichnung $\hat{\sigma}_{1}=-\sigma_{1}$

$$
\begin{aligned}
& \varrho_{0}=0, \\
& \hat{\sigma}_{1} \geqq\left|\varrho_{1}\right| \geqq 0 .
\end{aligned}
$$

Die Bedeutung von (16) kann mit Hilfe der differentiellen bedingten Wahrscheinlichkeiten dafür, daß das IT im Zeitintervall seine Geschwindigkeit $\pm c$ beibehält bzw. umkehrt, geklärt werden. Aus (6), (11) folgt

$p\left(\begin{array}{c|c} \pm c & \pm c \\ t & t+\Delta t\end{array}\right)=\left\{\begin{array}{r}1-\left(\hat{\sigma}_{1}-\varrho_{1}\right) c \Delta t+O_{t}^{2} \ldots++ \\ \left(\hat{\sigma}_{1}+\varrho_{1}\right) c \Delta t+O_{t}^{2} \ldots-+ \\ \left(\hat{\sigma}_{1}-\varrho_{1}\right) c \Delta t+O_{t}^{2} \ldots+- \\ 1-\left(\hat{\sigma}_{1}+\varrho_{1}\right) c \Delta t+O_{t}^{2} \ldots--\end{array}\right.$ 
Das IT behält also „fast immer“ seine Geschwindigkeit bei und wechselt die Richtung seiner Bewegung „fast nie“. Wenn (16) nicht gilt, hat das IT eine endlich große Wahrscheinlichkeit, die Richtung seiner Geschwindigkeit in einem beliebig kleinen Zeitbzw. Ortsintervall zu wechseln. Da es sich mit endlicher Geschwindigkeit $c$ bewegt, bleibt es in diesem Fal makroskopisch gesehen an ein und demselben Ort. Mit anderen Worten: Die Bedingung (16) garantiert die Diffusion des IT.

Man kann mit Hilfe von (16) zeigen, daß die Bahn des IT eine überall stetige und fast überall differenzierbare Kurve mit den konstanten Steigungen $\pm c$ ist. Die Gln. (18) können wegen der Markoff-2-Eigenschaft [vgl. (4) ] direkt integriert werden. Es gilt

$p\left(\begin{array}{c|c} \pm c & \pm c \\ t & t+\tau\end{array}\right)=\left\{\begin{array}{r}\exp \left\{-\left(\hat{\sigma}_{1}-\varrho_{1}\right) c \tau\right\} \ldots++ \\ 1-\exp \left\{-\left(\hat{\sigma}_{1}+\varrho_{1}\right) c \tau\right\} \ldots-+ \\ 1-\exp \left\{-\left(\hat{\sigma}_{1}-\varrho_{1}\right) c \tau\right\} \ldots+- \\ \quad \exp \left\{-\left(\hat{\sigma}_{1}+\varrho_{1}\right) c \tau\right\} \ldots--.\end{array}\right.$

Aus (19) folgt die mittlere Anzahl von Knicken pro Längeneinheit der Bahnkurve:

$$
n_{\mathrm{s}}=\hat{\sigma}_{1}\left(1-\left(\varrho_{1} / \hat{\sigma}_{1}\right)^{2}\right) \geqq 0 .
$$

Diese Beziehung läßt den ordnenden Einfluß einer äußeren Kraft $\left(\varrho_{1}\right)$ erkennen. Aus (13) folgen mit (11), (14), (15), (16) die Gleichungen

$$
\begin{aligned}
& \partial_{t} p\left(\begin{array}{l}
x \\
t
\end{array}\right)=-c \partial_{x} \pi_{r}, \\
& \partial_{t} \pi_{r}\left(\begin{array}{l}
x \\
t
\end{array}\right)=c\left(2 \varrho_{1}-\partial_{x}\right) p-2 c \hat{\sigma}_{1} \pi_{r}, \\
& \partial_{t} \pi_{a}\left(\begin{array}{l}
x \\
t
\end{array}\right)=-2 c \hat{\sigma}_{1} \pi_{a}+c\left(2 \varrho_{1}-\partial_{x}\right) p .
\end{aligned}
$$

Mit (2), (10) und den Bezeichnungen

$$
\tau=1 / 2 c \hat{\sigma}_{1}, \quad b=\left(\varrho_{1} / \hat{\sigma}_{1}\right) c, \quad D=c / 2 \hat{\sigma}_{1}
$$

ergibt sich aus $(21 \mathrm{~b}, \mathrm{c})$ eine Beziehung für den Wahrscheinlichkeitsstrom

$$
\tau \partial_{t} j+j=b p-D \partial_{x} p .
$$

Diese Beziehung kann als ein um den Trägheitsterm auf der linken Seite erweitertes 1. Ficksches Gesetz angesehen werden. Dieser Trägheitsterm wird erst durch die 2. Markoff-Stufe bedingt! Aus (23) folgt mit dem Erhaltungssatz für die Wahrscheinlichkeit (bzw. dem Erhaltungssatz für die Teilchenzahl oder dem Energiesatz)

$$
\partial_{t} p+\partial_{x} j=0
$$

die Gl. (E.4). Diese Gleichung ergibt sich natürlich auch direkt aus $(21 \mathrm{a}, \mathrm{b})$ durch Elimination von $\pi_{r}$. Die Gl. (E.4) beschreibt einen irreversiblen Prozeß. Das heißt, es gilt für alle Verteilungen $p\left(\begin{array}{l}x \\ t\end{array}\right)$, welche sich aus irgendeiner normierbaren Anfangsverteilung $p\left(\begin{array}{l}x \\ 0\end{array}\right)$ gemäß (E.4) entwickeln,

$$
\lim _{t \rightarrow \infty} p\left(\begin{array}{l}
x \\
t
\end{array}\right)=0 \ldots \text { alle } x \text {. }
$$

Diese Aussage kann mit Hilfe eines der Abelschen Grenzwertsätze nach Fourier-Laplace-Transformation von (E.4) leicht bewiesen werden. Wir geben noch das asymptotische Verhalten des Mittelwertes $E$ und der Streuung $S$ der Lagekoordinate sowie des Mittelwertes $E_{\mathrm{v}}$ und der Korrelationsfunktion $R_{\mathrm{v}}$ der Geschwindigkeit des IT an:

$$
\begin{array}{ll}
\lim _{t \rightarrow \infty} E(t) & \cong b t, \\
\lim _{t \rightarrow \infty} S(t) & \cong 2 D\left(1-\left(\varrho_{1} / \hat{\sigma}_{1}\right)^{2}\right), \\
\lim _{t \rightarrow \infty} E_{\mathrm{v}}(t) & =b, \\
\lim _{t \rightarrow \infty} R_{\mathrm{v}}\left(t, t+t_{0}\right) & =c^{2}\left(1-\left(\varrho_{1} / \hat{\sigma}_{1}\right)^{2}\right) e^{-t_{0} / \tau} .
\end{array}
$$

Die Parameter der Gl. (E.4), $D, b, \tau$ bzw. $c, \hat{\sigma}_{1}, \varrho_{1}$ können also experimentell bestimmt werden, wenn die mittlere Lage, die Streuung und die Korrelationsfunktion des IT gemessen werden können.

Läßt man die Anzahl $n_{s}$ der Knickpunkte pro Längeneinheit der Bahn des IT und gleichzeitig die Geschwindigkeit $c$ bei festen Werten von $b$ und $D$ über alle Grenzen wachsen, so strebt $\tau \rightarrow 0$ und (E.4) geht in eine Smoluchowski- bzw. FokkerPlanck-Gleichung über.

Führt man den Einstein-Smoluchowski-Limes (E.2) bei Markoff-Ketten 2. Stufe durch, so erhält man wieder Fokker-Planck-Gleichungen. Die 2. MarkoffStufe führt in diesem Fall nur zu gegenüber der 1. Stufe geänderten Werten der Beweglichkeit bzw. der Diffusionskonstanten.

Wir wollen zum Abschluß bemerken, daß eine höherer Stufe, nicht von besonderem Interesse zu sein scheint. Man würde dadurch nur die Möglichkeit erhalten, außer der gewöhnlichen Beschleunigung des IT die in der Mechanik praktisch bedeutungslosen höheren Beschleunigungen in den Formalismus einzuführen.

Der Verfasser dankt Herrn Prof. Dr. A. Stahl und seinem Kollegen Herrn Dr. D. v. BorRIEs für Diskus. sion. 